\title{
Transcriptome analysis reveals differentially expressed genes for improving survival of dormant Apostichopus japonicus in response to high temperature
}

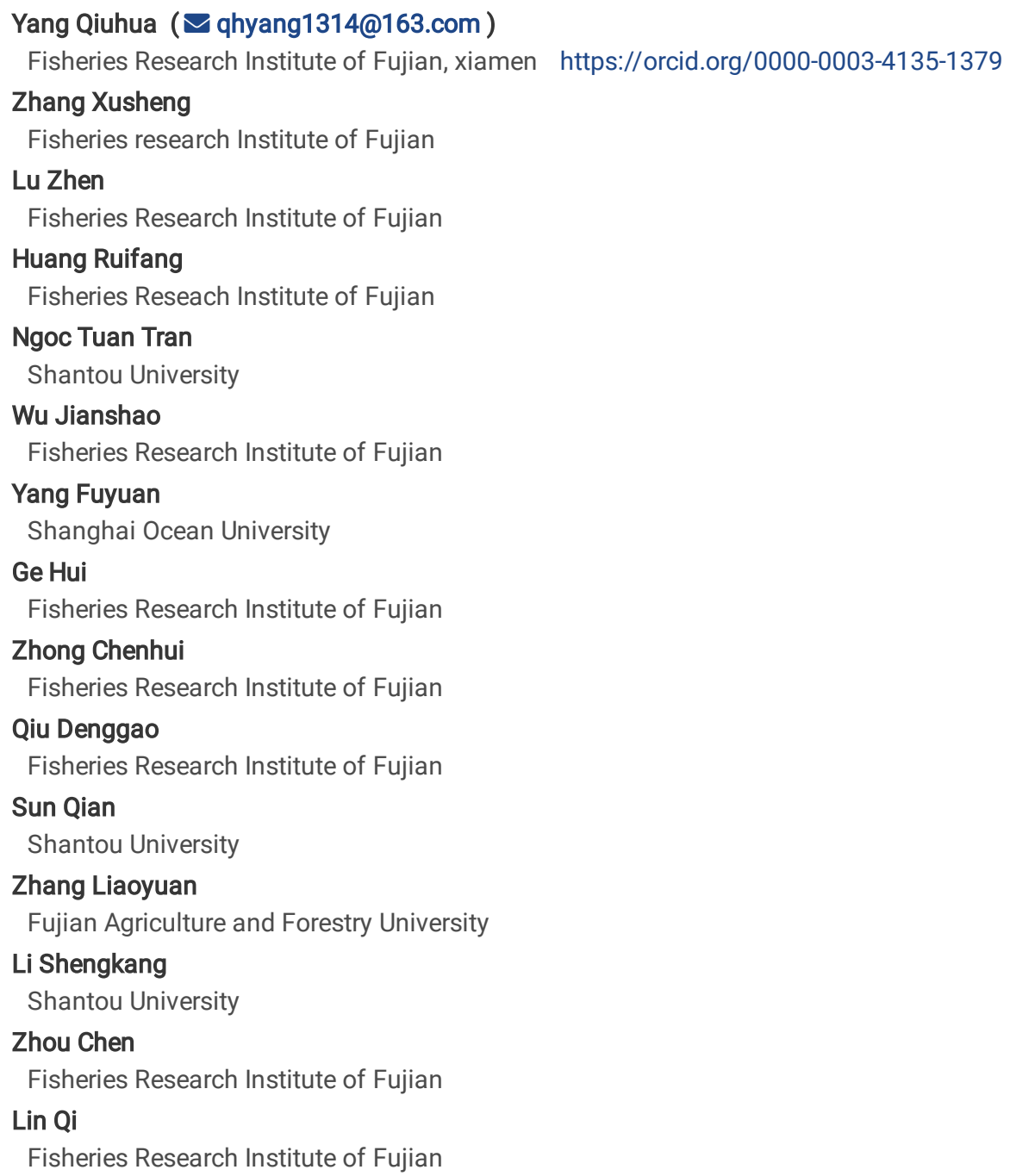

Research article

Keywords: Aestivation, Apostichopus japonicas, hypometabolism, fatty acid metabolism, carbohydrate hydrolysis, phagocytosis

Posted Date: November 21st, 2019

DOI: https://doi.org/10.21203/rs.2.17599/v1

License: (c) (i) This work is licensed under a Creative Commons Attribution 4.0 International License. Read Full License 


\section{Abstract}

Background: Aestivation is one of the strategies used by sea cucumbers (Apostichopus japonicas) in order to improve survival in response to the high-temperature and droughty conditions. Previous studies have carried out to investigate the immune or physiological alterations at the aestivation stage. However, it lacks information on the relationship between immunity and physiology. Herein, transcriptome sequencing was used to study gene expression during the aestivation stage. The results of this study provide a comprehensive understanding of the molecular mechanisms that protect sea cucumbers from the high-temperature condition, which favors improving survival in cultured sea cucumbers. Results: The transcriptome analysis of dormant (aestivation) and revival sea cucumbers generated 2,368 differentially expressed genes (down-regulation: 927; up-regulation: 1,441) and 39,081 unchanged genes. Basing on Gene Ontology and Kyoto Encyclopedia of Genes and Genomes enrichment analyses, the down-regulated genes of dormant group were identified to be involved in DNA replication, RNA metabolic process, protein modification and biosynthesis, macromolecule metabolism, and cellular metabolism, which resulted in the inhibition of motility, skeletal development, neural activity, cell proliferation and development of A. japonicas. In contrast, the up-regulated genes were associated with fatty acid metabolism, carbohydrate hydrolysis, and phagocytosis. Protein-protein interaction network analysis further revealed that carbohydrate hydrolysis promoted the phagocytosis activity in the dormant group. Furthermore, the expression pattern of all tested genes in qRT-PCR analysis fitted well with those in RNASeq, with the exception of FASNL, which was unchanged in the qRT-PCR but up-regulated in RNA-seq. Conclusions: During the dormant stage, sea cucumbers decreased DNA replication, transcription and translation to achieve a hypometabolic state beneficial for reducing energy consumption On the contrary, fatty acid metabolism and carbohydrate hydrolysis were increased for energy supply. Moreover, high levels of carbohydrate hydrolysis promoted phagocytosis, which is a crucial innate immune response to infection by pathogens. These results provided new insight into potential molecular mechanisms that enable the sea cucumbers to respond to high temperatures. Keywords: Aestivation, Apostichopus japonicas, hypometabolism, fatty acid metabolism, carbohydrate hydrolysis, phagocytosis

\section{Background}

Sea cucumber (Apostichopus japonicas) is echinoderms from the family Holothuroidea (phylum Echinodermata). It is well known that several species of sea cucumbers are detritus feeders which enable them to ingest sediments (like organic matter, bacteria, and protozoa) for acquiring nutrients. Thus, $A$. japonicas have been shown to be very important for maintaining environmental cleaning [1]. Moreover, because of the advantages of $A$. japonicas containing an abundance of collagen and mucopolysaccharides and high values in medicine and commerce [2, 3], thus they are widely cultured in many countries such as Japan, Korea, and China [4].

In China, A. japonicas is an important commercial mariculture species, which mainly distributed along the coastal region of the north in the past years. Most of the previous studies have only focused on studying this species distributing in northern China $[4,5]$. In recent years, sea cucumbers have been cultured popularly in southern China [6-8]. Previous studies showed that different-regional culture environments significantly affected the metabolism and immunity of sea cucumbers $[9,10]$. Thus, the metabolisms and immune responses of the $A$. japonicas cultured in southern China may differ from that cultured in the north. This is due to differences in the climate conditions between the two regions, the higher temperature was in southern China, thus, the study efforts on studying the behaviors (like aestivation) of sea cucumbers cultured in southern China are significant.

Aestivation is a common strategy for both vertebrates and invertebrates to maintaining the survival suffering from high-temperature and arid conditions [11]. During the summer period, the high temperature (over $25^{\circ} \mathrm{C}$ ) induced the sea cucumber entering the aestivation state [4]. Furthermore, the temperature of over $20^{\circ} \mathrm{C}$ provided a favorable condition for microorganism multiplication, which cause a large-scale outbreak of diseases and severely threaten the survival of multicellular organisms [12-15]. Therefore, the aestivation of sea cucumber also is a protective strategy in resistance to pathogens via improving the expression of immune genes, including lysozyme, C-type lectin, and complement factors [4, 5, 16]. However, the metabolism and immunity of dormant sea cucumber were separately carried out in the previous studies, little is known about relationships between metabolism and immunity $[4,5,8,17]$. In this study, transcriptome sequencing was used to wholly reflect the molecular response of the $A$. japonicas distributing in southern China during the summer aestivation stage.

As shown in many studies, high-throughput RNA-Sequencing (RNA-Seq) technology is an excellent approach to study the transcriptional levels of large numbers of genes in tissues or organisms. The results of sequencing data can provide the "whole picture" of the gene expression profile in the organisms [18-21]. Obtained genes were annotated upon Protein family (Pfam), Superfamily, Gene Ontology (GO) and Kyoto Encyclopedia of Genes and Genomes (KEGG) database for characterizing their functions at the molecular and cellular levels [22-25]. In order to understand the potential molecular mechanism relating to the aestivation stage of sea cucumber, differentially expressed genes (DEGs) were analyzed to elucidate the functional categories based on the GO and KEGG enrichment analyses [22, 23]. Moreover, Venn diagram analysis was used to study the special expression of genes in each group, and then performed GO and KEGG enrichment to analyze functional categories [5, 22]. Therefore, the database of Search Tool for the Retrieval of Interacting Genes (STRING) was used to study functional interaction of proteins (including stable complexes, metabolic pathways and a bewildering array of direct and indirect regulatory interactions), which was also usually used to analyze the transcriptomic profile $[19,26]$. Therefore, our study employed transcriptome sequencing to investigate the alteration of physiological metabolism and immune in the aestivation stage, and then analyze the potential associations between them. The results of this study provide new insight into the potential molecular mechanisms of physiological metabolisms and immune responses, which are crucial in improving the survival of sea cucumber in aquaculture. 


\section{Results}

\section{Transcriptome quantification}

A total of 474,876,912 raw reads were acquired after RNA-seq containing 457,223,622 clean reads ( $96.28 \%$ of the raw reads) after removing adaptor sequences, reads containing poly-N and low-quality reads (Table 1$)$. Moreover, 301,336,131 reads (65,90\% of the clean reads) were mapped to the reference genome of $A$. japonicus which was composed of $279,721,413(61.18 \%$ of the clean reads) uniquely mapped reads and $21,614,718$ multiply mapped reads (4.73\% of the clean reads). The average Q20 and Q30 of the read data was $97.43 \%$ and $92.90 \%$, respectively. The QC content was accounted for $41.18 \%$ of the sequencing data.

\section{Differential expression genes (DEGs) characterization}

A total of 2,368 DEGs (including 927 down-regulated and 1,441 up-regulated genes) and 39,081 unchanged genes were acquired in the dormant group compared to the revival group (Fig. 1). In the dormant group, GO analysis revealed that the down- and up-regulated genes were annotated to sub-categories under three primary categories (i.e. biological process, cellular component, and molecular function) (Fig. 2). The down-regulated genes were assigned to 41 sub-categories, which were mainly classified into DNA replication, RNA metabolic process, protein modification and biosynthesis, macromolecule metabolism, and cellular metabolism (Fig. 2A). By contrast, the up-regulated genes were assigned to 85 subcategories, such as peptidase activity, acting on L-amino acid peptides, peptidase activity, proteolysis, endopeptidase activity, metallopeptidase activity, serine-type endopeptidase activity, serine-type peptidase activity, and serine hydrolase activity (all belongs to protein degradation category). Moreover, the carbohydrate metabolic process was also significantly enriched in the dormant group (Fig. 2B).

To better understand the function of DEGs in the dormant group, an analysis of the KEGG enrichment using was performed (Fig. 3). The results showed that the down-regulated genes that related to the gene expression process (i.e. spliceosome, RNA transport, ribosome biogenesis in eukaryotes and ribosome) and proteasome metabolism pathways were significantly enriched (Fig. 3A). The up-regulated genes were identified to associate with the pathways relating to phagocytosis (lysosome, endocytosis, and phagosome), lipid metabolism (fatty acid metabolism, steroid biosynthesis, ether lipid metabolism, and glycerophospholipid metabolism), and amino acid metabolism (glycine, serine and threonine metabolism, arginine and proline metabolism, tyrosine metabolism, and histidine metabolism) (Fig. 3B). Otherwise, the metabolisms relating to carbohydrates (including starch and sucrose metabolisms) were also enhanced in the dormant group (Fig. 3B).

\section{Expression of genes between dormant and revival groups}

In the Venn diagram analysis, a number of 1,850 or 405 genes were only expressed in the dormant or revival groups, respectively. All of these genes were further analyzed basing on the KEGG database. The results revealed that the pathways involving in the carbohydrate metabolisms (i.e. starch and sucrose metabolism and other glycan degradation), fatty acid-related metabolism (fatty acid metabolism, biosynthesis of unsaturated fatty acids, and fatty acid elongation) and phagocytosis (phagosome, lysosome, and endocytosis) were significantly enriched in the dormant group compared to the revival group.

\section{Protein-protein interaction (PPI) analysis of up- and down-regulated genes}

The STRING database was used to analyze the interactions among different expression genes and the results on the characterization of genes in the PPI diagram, which were shown in Table S1A. The top core down-regulated genes were classified into the transcription and translation, such as methyltransferase NSUN6, ribosomal protein uS7, translation initiation factor eIF4A II, ribosome production factor 1, RNA cytidine acetyltransferase, U3 small nucleolar ribonucleoprotein protein IMP4, pre-rRNA-processing protein TSR1-like, Eukaryotic translation initiation factor 3 subunit B, GMP synthase, and eukaryotic translation initiation factor 6 (Fig. 6A). Whist other top down-regulated genes involved in decreasing motility (dystrophinlike protein, ubiquitin carboxyl-terminal hydrolase 19, zinc finger MYND domain-containing protein 10), skeletal development (synaptotagmin-7 and twisted gastrulation protein), neural activity (synaptotagmin-7 and vesicular glutamate transporter 1, and choline 0-acetyl-transferase), cell proliferation (Baculoviral IAP repeat-containing protein 2 and ubiquitin carboxyl-terminal hydrolase 19) and development (twisted gastrulation protein, intraflagellar transport protein 27, and SPARC-related modular calcium-binding protein 1) in the dormant group.

In contrast, the interactions among the up-regulated genes were found to mainly relate to the carbohydrate hydrolysis (including maltaseglucoamylase, hexokinase-1, pancreatic alpha-amylase, alpha-amylase $\mathrm{B}$, alpha-amylase) and the promotion of phagosome $\left(\mathrm{V}\right.$-type $\mathrm{H}^{+}$-transporting ATPase subunit a) (Fig. 6B). These metabolic pathways that up-regulated genes in the PPI analysis were also consistent with KEGG enrichment of DEGs and Venn diagram analysis. The detailed characterization of related genes was shown in Table S1.

\section{Pathway analysis}

The pathways found in the dormant group were elucidated in detail in Fig. 7. Moreover, the functional characterization and gene expression (basing on TPM value) were shown in Table 2. In the dormant group, the high expression of genes, including fatty acid synthase (FASN, 2.86-fold), fatty acid synthase-like (FASNL, 3.15-fold), very long-chain specific acyl-CoA dehydrogenase (ACADVL, 1.13-fold), long-chain specific acyl-CoA dehydrogenase (ACADL, 2.83-fold) and malonyl-CoA-acyl carrier protein transacylase (MCAT, 1.23-fold), relating to the pathways of fatty acid biosynthesis and 
degradation were observed. A high level of fatty acid biosynthesis and degradation promoted the production of acetyl-CoA, a substrate used in the TCA cycle. Moreover, the genes hexokinase-1 (HK1, 1.28-fold), pancreatic alpha-amylase (PAMY, 7.90-fold), alpha-amylase B (AMYB, over 10-fold), alpha-amylase (AMY, 2.86-fold), and maltase-glucoamylase (MGAM, 6.33-fold) that related to carbohydrate degradation were highly expressed in the dormant group. The carbohydrate degradation also promoted the production of acetyl-CoA and TCA cycle. Basing on the PPI analysis, the upregulation of PAMY, AMYB, AMY stimulated the expression of MGAM and HK1. The expression of HK1 bond to UBCV, which enhanced the phagocytosis, therefore, interacted with VATPA (2.06-fold). Furthermore, the up-regulation of phagocytosis-related genes (i.e. actin-related protein 2/3 complex subunit 5A (ARPC5A, 1.46-fold), actin-related protein 2/3 complex subunit 5B (ARPC5B, 2.31-fold), PH and SEC7 domain-containing protein (PSD, 1.11-fold), N-acetylglucosamine-6-sulfatase (GNS, 1.18-fold), N-acetylgalactosamine-6-sulfatase-like (GNSL, 2.99-fold) and macrophage mannose receptor 1 (MRC1, 3.30-fold) were associated with the scavenging of pathogen in the dormant $A$. japonicus.

\section{qRT-PCR analysis}

Eighteen genes relating to the pathways in Fig. 7 were used to validate using qRT-PCR (Fig. 8). The results revealed that with the exception of FASNL, which was slightly down-regulated, all tested genes related to phagocytosis, fatty acid metabolism, and carbohydrate hydrolysis were increased in the dormant group. The relative expression of genes demonstrated that 17/18 (94\%) genes showed a similar trend compared to those in the RNAseq. These results indicated that the data obtained from RNA-seq is highly reliable. Basing on the RNA-seq, AMYB was detected in the dormant group but not in the revival group. The TPM value ratio (dormant group to revival group) was infinite, which is hard to exhibit in graphics. Thus, the biggest value of ten TPM value was used to replace infinity for showing high expression of AMYB in the dormant group (Fig. 8).

\section{Discussion}

The increase in summer temperature facilitates the disease outbreaks caused by microorganisms, which threaten the survival of marine organisms $[12,13]$. The higher temperature in lower latitudes usually causes worse effects on the animals [15]. To protect against pathogen infections, sea cucumbers had evolved an aestivation behavior in response to high temperature $[4,5,20]$. As previously comparable reports, most studies of $A$. japonicas were carried out in northern China $[4,5,17,28]$. However, the changes of metabolisms and immune responses of dormant $A$. japonicas adapting to climate warming were scarcely reported. In this study, the transcriptome analysis was performed to investigate the gene expression of $A$. japonicas in warmer southern China (Fujian province), which is important in exploring the adaption mechanism of $A$. japonicas in response to the higher temperature.

Totally, 2,368 DEGs (down-regulation: 927 and up-regulation: 1,441) were found in the dormant group compared with the revival group. The enrichment analysis of down-regulated genes revealed that $41 \mathrm{GO}$ terms and 20 KEGG pathways were identified to be associated with the DNA, RNA, protein, and cellular metabolisms in the dormant group. These results implied that the physiological functions were decreased in the dormant group, and furthermore leading to the inhibition of growth and development of $A$. japonicas during the aestivation period. These results are similar to the previous studies that organisms reduced metabolisms of DNA, RNA, and protein during the dormant period, which further affected the growth and development $[4,29,30]$. In contrast, the up-regulated genes related to protein degradation, phagocytosis, carbohydrate metabolism, and fatty acid metabolism were identified through the GO and KEGG analyses. Basing on the KEGG database, long-chain fatty acids and saccharides are able to produce acetyl-CoA, which promotes the synthesis of ATP through the TCA cycle. The previous studies on the sea cucumbers cultured in northern China also acquired similar results that carbohydrate catabolism and fatty acid degradation for energy supplying were increased during the aestivation [4, 20,30,31]. These results indicated that the dormant animals need to maintain energy supplying via the increasing consumption of stored carbohydrate and fatty acids as the food shortage [11]. Moreover, phagocytosis-related pathways (i.e. endocytosis, lysosome, and phagosome) were up-regulated in dormant sea cucumbers, which may play an important role in the innate immunity as a primary defense in the invertebrates [32-37]. Among these phagocytosis-related genes, C-type lectin genes (macrophage mannose receptor 1-MRC1) was significantly upregulated (3.3-fold) in the dormant group. Previously, the up-regulation of the lysosome and C-type lectin were observed in the dormant sea cucumbers [4]. In the Venn diagram analysis, 2,255 specially expressed genes were obtained. The KEGG enrichment of these genes revealed that the phagocytosis (i.e. endocytosis, lysosome, and phagosome), as well as fatty acid and carbohydrate metabolism were altered in the dormant group, compared to that in the revival group. Therefore, the hypometabolism mainly occurred, but the fatty acid metabolism, carbohydrate metabolism, and phagocytosis activity were increased during the aestivation stage in sea cucumbers.

In the subsequent protein-protein interaction (PPI) analysis, in the dormant sea cucumbers, the core down-regulated genes (methyltransferase NSUN6, ribosomal protein, translation initiation factor, ribosome production factor 1, RNA cytidine acetyltransferase, U3 small nucleolar ribonucleoprotein protein, pre-rRNA-processing protein, eukaryotic translation initiation factor 3 subunit B, GMP synthase, and eukaryotic translation initiation factor 6) were assigned to the transcriptional and translational regulations [38]. Previously, the decreased levels of transcriptional and translational gene expression were also observed during the dormant stage of northern sea cucumber and other animals [4, 30, 39]. Once the animals enter the aestivation or hibernation states, slow movements decrease their opportunity to acquire adequate food. The food shortages cause energy deficiency for the body; the animals reduce the levels of metabolisms and energy consumption, leading to the inhibition of most of the gene expression and protein translation activities [40]. Furthermore, these down-regulated genes regulate the motility, skeletal development, neural activity, 
cell proliferation and development of $A$. japonicas according to Uniprot database analysis [41]. Our findings are consistent with those of other studies and suggested that the sea cucumber reduced the expression of transcriptional and translational genes relating to the hypometabolism (e.g., decreased motility, stopped skeletal development, declined neural activity, and reduced cell proliferation and development) [4, 39, 40]. This mechanism is accordant to reducing energy consumption in response to the absence of energy intake during the aestivation of sea cucumber at high temperature $[2,39]$.

However, the PPI analysis showed that the up-regulated genes in the dormant sea cucumbers were identified as the carbohydrate hydrolase, including pancreatic alpha-amylase (PAMY), alpha-amylase B (AMYB), alpha-amylase (AMY), maltase-glucoamylase (MGAM) and hexokinase-1 (HK1) [42-45]. Basing on STRING database, the HK1 interact with the ubiquitin C variant (UBCV), then improved the phagosome activity via increasing the expression of V-type H+-transporting ATPase subunit A (VATPA), which was ATP-dependent proton pumps responsible for acidification of phagosome [46]. These results suggested that the up-regulation of carbohydrate hydrolase promoted the phagocytosis in the dormant $A$. japonicas. In addition, expression of other phagocytosis-related genes (actin-related protein 2/3 complex subunit 5A (ARPC5A), actinrelated protein 2/3 complex subunit 5B (ARPC5B), PH and SEC7 domain-containing protein (PSD), N-acetylglucosamine-6-sulfatase (GNS), Nacetylgalactosamine-6-sulfatase-like (GNSL), and macrophage mannose receptor 1 (MRC1)) were also increased in the dormant $A$. japonicas. Similar to previous studies, immune cells recognized pathogens via pattern recognition receptors in combination with pathogen-associated molecular patterns (PAMPs) [47]. MRC1 is a typical pattern recognition receptor, which ligates with the mannose-rich glycoconjugates of pathogens $[48,49]$. The up-regulation of MRC1 indicated that the immune cells improved the recognition of pathogens in dormant $A$. japonicas, promoting the phagocytosis latterly. The increased expression of ARPC5A, ARPC5B, and PSD promoted the phagocytosis via forming vesicles for trafficking pathogen molecular to the phagosome, a crucial organelle in scavenging pathogens [16, 50-52]. Otherwise, the up-regulation of lysosomal GNS and GNSL accelerated the hydrolysis of heparan sulfate, which is important in responses to viral infection. The heparan sulfate was also found to be a crucial material in triggering of autoimmune in the immune cells. These alterations effectively limited pathogen infections [53-57]. Moreover, the previous studies carried out in sea cucumbers also acquired a similar result that carbohydrate metabolism, lysozyme, and phagocytosis were upregulated during the aestivation stage [4]. Thus, in the dormant $A$. japonicas, the high levels of carbohydrate metabolism promoted the phagocytosis activity, which plays a crucial role in scavenging pathogens and improving survival at seasonal high temperatures.

Furthermore, the TPM value was used to characterize the pathway alterations [58]. In the dormant A. japonicas, high expression of PAMY, AMYB, AMY, and MGAM promoted starch hydrolysis to produce glucose, which is transferred into the TAC cycle via glycolysis pathway [42-45]. An upregulation of ACADVL and ACADL in the dormant $A$. japonicas degraded long-chain fatty acids to acetyl-CoA, which was an essential material involved in the TCA cycle that is used for ATP synthesis $[59,60]$. A similar finding was observed in a previous study that starch and fatty acids hydrolase were highly expressed during the deep-aestivation of sea cucumbers [4]. These results suggested that the degradation of fatty acids and starch was the main route for providing energy in the dormant sea cucumbers. In addition, the HK1 interacts with VATPA, which then accelerates with $\mathrm{H}^{+}$and they together enter the phagosome, attending to improve phagocytic activities [46]. Thus, the high expression of HK1 indicates its roles in improving the immune system of sea cucumbers during the aestivation period. Moreover, the phagocytosis-related genes (ARPC5A, ARPC5B, PSD, GNS, GNSL, and MRC1) were up-regulated in the dormant group. This result is consistent with the previous results that an up-regulation of carbohydrate hydrolase not only catalyzes starch degradation but also promotes phagocytosis activity. Optimal carbohydrate diets have significantly stimulatory effects on phagocytosis $[61,62]$. Therefore, our results indicated that the dormant $A$. japonicas consumed carbohydrate and fatty acids for energy supply. High levels of carbohydrate metabolism improved the expression of phagocytosis-related genes beneficial for resistance to pathogens during the aestivation of $A$. japonicas. However, the above-mentioned results were only based on transcriptome analysis of the RNA level, it is needed to investigate in detail in the future to validate the findings in this current study.

\section{Conclusions}

In this study, the transcriptome analysis was used to explore potential molecular mechanisms in the dormant $A$. japonicas. Compared with the revival group (RG), a total of 2,368 DEGs were identified in the dormant group (DG). Basing on GO and KEGG analysis, the down-regulated genes in DG were mainly annotated to DNA, RNA, and protein metabolic process. PPI analysis further revealed that the down-regulation of DNA, RNA, and protein metabolic process reduced the motility, skeletal development, neural activity, cell proliferation, and development of $A$. japonicas. In contrast, the up-regulated genes were functionally classified into fatty acid and carbohydrate metabolisms. In the dormant $A$. japonicas, a high level of carbohydrate hydrolysis promoted the phagocytosis, which was a primary innate immune response in the invertebrates. Thus, the improvement of phagocytosis facilitates the $A$. japonicas increased their resistant ability against the pathogen invasion during the aestivation stage. The findings of this study provided new insights into the potential mechanisms that $A$. japonicas are able to change their physiological response in order to respond to the high temperature and pathogen invasion at the molecular level.

\section{Methods}

\section{Sample collection and cDNA library construction}

The healthy A. japonicus individuals were obtained from Dongshan base of Fujian Fisheries Research Institution (Zhangzhou, Fujian, China), and acclimatized in Fujian Fisheries Research Institution $\left(24^{\circ} 29^{\prime} 6.92^{\prime \prime} \mathrm{N}, 118^{\circ} 04^{\prime} 32.49^{\prime \prime} \mathrm{E}\right)$ for 4 month. About $5 \mathrm{~g}$ of each individual was sampled in 
August, September, and October 2019. The sea cucumbers collected in August and September were named as the dormant group (DG), and samples from October were named as revival group (RG). Each group contained at least three replications. The samples were collected and immediately kept into liquid nitrogen for RNA extraction.

The sea cucumber samples were ground in a ceramic pestle. The total RNA was extracted using Trizol Kit (Invitrogen, USA) following the manufacturer's instructions. The quality and quantity of total RNA were also estimated using $1 \%$ gel electrophoresis, Nano Photometer spectrophotometer (IMPLEN, CA, USA), and Agilent Bioanalyzer 2100 system (Agilent Technologies, CA, USA). The obtained total RNA was used to synthesize the cDNA libraries using the PrimeScript RT reagent Kit with gDNA Eraser (Takara, Dalian, China) following the manufacturer's instructions. The cDNA was used as the templates for qRT-PCR and RNA sequencing (RNA-Seq) transcriptome.

\section{Illumina RNA sequencing}

At least $1 \mu \mathrm{g}$ of total mRNA was used to construct cDNA libraries. The purified mRNA from total RNA was cut into fragments by using the NEBNext ${ }^{\circledR}$ Ultra ${ }^{\text {TM }}$ RNA Library Prep Kit (Illumina, USA) for constructing the cDNA library. Put simply, oligo (dT) magnetic beads enriched mRNA with polyA, firstly. NEB fragmentation buffer was used to randomly fragment the mRNA into small fragments, which was then used as templates for synthesizing the first-strand cDNA using random hexamer primer and M-MuLV Reverse Transcriptase. Subsequently, DNA Polymerase I and RNase $\mathrm{H}$ were used to produce the second-strand cDNA with dNTPs. Double-strand cDNA was purified accepted end repair, A-base addition, and ligation of the Illumina-indexed adaptors. Finally, 150 200 bp cDNA was used for the PCR amplification and the PCR products were purified using AMPure XP beads (Beckman Coulter, Beverly, USA), which was used to generate cDNA libraries. The cDNA library quality was further assessed using the Agilent Bioanalyzer 2100 system. The concentration of cDNA over $2 \mathrm{nM}$ was used to sequence by an Illumina Hiseq ${ }^{\mathrm{TM}} 2500$ platform.

\section{Sequence assembly and gene functional annotation}

Quality control was performed removing adaptor sequences, reads containing poly-N and low-quality reads, then calculated the Q20 and Q30 of clean reads. HISAT2 v2.0.5 software was used to further aligned and obtained the clean reads to reference the genome of $A$. japonicus that the accession number is PRJNA354676 [27]. The novel transcript assembly was performed using StringTie (version v1.3.3b) and these transcripts were annotated basing on Protein family (Pfam), Superfamily, Gene Ontology (GO) and Kyoto Encyclopedia of Genes and Genomes (KEGG) database. The FeatureCounts (v1.5.0-p3) was used to count read numbers of each gene. Basing on the gene length and read count, FPKM (Fragments Per Kilobase of transcript per Million mapped reads) of each gene was further calculated. The subsequent fold-change calculated were based on the FPKM ratio between dormant and revival groups. DESeq2 was used to identify the genes with $P$-value $<0.05$ and $\mid$ log 2 (foldchange) $\mid>1$ as differently expressed genes (DEGs).

GO enrichment of DEGs was performed with clusterProfiler R package and corrected the bias of gene length. For better understanding the functions of the genes in a biological system, KEGG enrichment was also carried out with clusterProfiler R package, which well-explained the genes at molecular levels. Moreover, the specific expression of genes in dormant and revival groups was analyzed and exhibited in a Venn diagram. Specific genes were also performed with GO and KEGG enrichment.

\section{Protein-protein interaction (PPI) analysis}

Protein-protein interaction analysis of DEGs was carried out using the STRING database (http://string-db.org/), which investigated the direct and indirect associations among different proteins. The networks showing up- and down-regulated genes were drawn using Cytoscape software based on the results of STRING analysis.

\section{Quantitative real-time PCR (qRT-PCR) analysis of metabolism-related genes}

To validate the results of gene expression obtained from the transcriptomic sequencing, quantitative real-time PCR (qRT-PCR) was performed to detect the gene expression using the SYBR ${ }^{\circledR}$ Premix Ex Taq ${ }^{\mathrm{TM}}$ II Kit (Takara, Dalian, China) in LightCycler ${ }^{\circledR} 480$ (Roche, USA). Total RNA from $A$. japonicus was extracted using Total RNA kits $\otimes($ Omega, USA) following the manufacturer's instructions. cDNA was synthesized using PrimeScript RT reagent Kit with gDNA Eraser (Takara, Dalian, China) following the manufacturer's instructions. The obtained cDNA was used as templates for qRT-PCR. In this study, 18 genes relating to carbohydrate metabolism, fatty acid metabolism, TCA circle, and phagocytosis pathways were used in the qRT-PCR. A $20-\mu \mathrm{L}$ reaction mixture contained $10 \mu \mathrm{L}$ of SYBR ${ }^{\circledR}$ Premix Ex Taq ${ }^{\mathrm{TM}}$ II, $2 \mu \mathrm{L}$ of cDNA, $0.8 \mu \mathrm{L}$ (10 mM) of each (forward and reverse) primer (Table 3), and $6.4 \mu \mathrm{L}$ of $\mathrm{ddH}_{2} \mathrm{O}$. The amplification was run with the followed program: denaturation at $95^{\circ} \mathrm{C}$ for $30 \mathrm{~s}$, followed by 40 cycles of $95^{\circ} \mathrm{C}$ for $5 \mathrm{~s}, 60^{\circ} \mathrm{C}$ for $20 \mathrm{~s}$, and melting curve analysis from $65^{\circ} \mathrm{C}$ to $95^{\circ} \mathrm{C}$. Each sample was done in triplicate. The relative expression of selected genes was calculated using the $2^{-\Delta \Delta \mathrm{Ct}}$ algorithm normalized to the internal control ( $\beta$-actin).

\section{Abbreviations}


Pfam: Protein family

GO: Gene Ontology

KEGG: Kyoto Encyclopedia of Genes and Genomes

DEGs: Differentially expressed genes

STRING: Search Tool for the Retrieval of Interacting Genes

PPI: Protein-protein interaction

TPM: Transcripts per million

FPKM: Fragments per kilobase of transcript per million mapped reads

qRT-PCR: Quantitative real-time PCR

RNA-seq: RNA sequencing

ARPC5A: Actin-related protein 2/3 complex subunit 5A

ARPC5B: Actin-related protein 2/3 complex subunit 5B

PSD: PH and SEC7 domain-containing protein

GNS: N-acetylglucosamine-6-sulfatase

GNSL: N-acetylgalactosamine-6-sulfatase-like

VATPA: V-type $\mathrm{H}^{+}$-transporting ATPase subunit A

MRC1: Macrophage mannose receptor 1

HK1: Hexokinase-1

PAMY: Pancreatic alpha-amylase

AMYB: Alpha-amylase B

AMY: Alpha-amylase

MGAM: Maltase-glucoamylase

FASN: Fatty acid synthase

FASNL: Fatty acid synthase-like

ACADVL: Very long-chain specific acyl-CoA dehydrogenase

ACADL: Long-chain specific acyl-CoA dehydrogenase

MCAT: Malonyl-CoA-acyl carrier protein transacylase

UBCV: Ubiquitin $\mathrm{C}$ variant

\section{Declarations}

\section{Availability of data and materials}

All original sequence data in this study were submitted to the NCBI Sequence Read Archive database (https://www.ncbi.nlm.nih.gov/bioproject/PRJNA589138) under accession number PRJNA589138.

\section{Ethics declarations}

Ethics approval and consent to participate 
All animal handling procedures were reviewed and approved by the ethics committee of the "Regulations for the Administration of Affairs Concerning Experimental Animals". The Institutional Animal Care and Use Committee of Fisheries Research Institute of Fujian, China, approved the experiments.

\section{Consent for publication}

All the co-authors consent to publish the work in BMC Genomics.

\section{Competing interests}

The authors declare that they have no competing interests.

\section{Funding}

This work was supported by the specialized research foundation of Fujian provincial department of science \& technology, China (Grant No. 2018R1003-1 and 2019R1013-5), and the project of marine fisheries of Fujian provincial department of ocean and fisheries, China (Grant No. 2020HYJG02 and FJHJF-L-2020-4). These funding bodies had no role in study design, analysis, decision to publish, or preparation of the manuscript.

\section{Author information}

Qiuhua Yang and Xusheng Zhang contributed equally to this work.

\section{Affiliations}

Key Laboratory of Cultivation and High-value Utilization of Marine Organisms in Fujian Province, Fisheries Research Institute of Fujian, Xiamen, 361013, China

Qiuhua Yang, Xusheng Zhang, Zhen Lu, Ruifang Huang, Jianshao Wu, Fuyuan Yang, Hui Ge, Chenhui Zhong, Denggao Qiu, Chen Zhou \& Qi Lin Guangdong Provincial Key Laboratory of Marine Biology, Marine Biology Institute, Shantou University, Shantou, 515063, China

Qiuhua Yang, Xusheng Zhang, Ngoc Tuan Tran, Qian Sun \& Shengkang Li

College of Life Sciences, Fujian Agriculture and Forestry University, Fuzhou, 350002, China

Liaoyuan Zhang

\section{Contributions}

YQH designed, supervised the research. ZXS performed most of the experiments and wrote the paper with assistance from NTT, ZLY, LZ, HRF, WJS, YFY, GH, ZCH, SQ, QDG, LSK, ZC and LQ. All authors made contribution to the final version of this manuscript. All authors read and approved the final manuscript.

\section{Corresponding authors}

Correspondence to Liaoyuan Zhang, Shengkang Li or Qi Lin.

\section{Acknowledgements}

Not applicable.

\section{References}

1. Yang H, Zhou Y, Zhang T, Yuan X, Li X, Liu Y, et al. Metabolic characteristics of sea cucumber Apostichopus japonicus (Selenka) during aestivation. J Exp Mar Biol Ecol. 2006;330(2):505-510.

2. Ji TT, Dong YW, Dong SL. Growth and physiological responses in the sea cucumber, Apostichopus japonicus Selenka: Aestivation and temperature. Aquaculture. 2008;283:180-187.

3. Duan X, Zhang M, Mujumdar AS, Wang SJ. Microwave freeze drying of sea cucumber (Stichopus japonicus). J FOOD ENG. 2010;96:491-497.

4. Zhao Y, Yang HS, Storey KB, Chen MY. RNA-seq dependent transcriptional analysis unveils gene expression profile in the intestine of sea cucumber Apostichopus japonicus during aestivation. Comp Biochem Phys. 2014;10:30-43.

5. Li C, Fang H, Xu D: Effect of seasonal high temperature on the immune response in Apostichopus japonicus by transcriptome analysis. Fish Shellfish Immunol. 2019;92:765-771. 
6. Fang LQ, Hu Gi; Yang QH; Lin Q; Zhou C; Li SJ. Cloning and Characterization of Serum Amyloid A (SAA) from Sea Cucumber, Apostichopus japonicus. Nanosci Nanotech Let. 2017;9(5):785-791.

7. Yu ZH, Zhou Y, Yang HS, Hu CQ. Bottom culture of the sea cucumber Apostichopus japonicus Selenka (Echinodermata: Holothuroidea) in a fish farm, southern China. Aquac Res. 2014;45:1434-1441.

8. Yu ZH, Zhou Y, Yang HS, Ma YE, Hu CQ. Survival, growth, food availability and assimilation efficiency of the sea cucumber Apostichopus japonicus bottom-cultured under a fish farm in southern China. Aquaculture. 2014;426-427:238-248.

9. Wu ZC, Chen HW, Wang WL, Jia B, Yang TL, Zhao ZF, et al. Differentiation of dried sea cucumber products from different geographical areas by surface desorption atmospheric pressure chemical ionization mass spectrometry. J Agric Food Chem. 2009;57:9356-9364.

10. Mercedes GW, Roggatz CC, Rodrigues MJ, Barreira L, Manuela MDS , Custódio, L. A new insight into the influence of habitat on the biochemical properties of three commercial sea cucumber species. Int Aquat Res. 2018;10(4):361-373.

11. Kenneth B, Storey JMS. Aestivation: signaling and hypometabolism. J Exp Biol. 2012;215:1425-1433.

12. Lacoste AFJ, Malham SA. Cueff F, Gélébart CC, Lange M, Poulet SA. A Vibrio splendidus strain is associated withsummer mortality of juvenile oysters Crassostreagigasin the Bay of Morlaix (North Brittany, France). Dis Aquat Org. 2001;46: 139-114.

13. Sonia A, Richard SO, Pieter TJ, Johnson SK, Harvell CD. Climate Change and Infectious Diseases: From Evidence to a Predictive Framework. Science. 2013;341(6145):514-519.

14. Elliot S, Blanford S, Thomas M. Host-pathogen interactions in a varying environment: Temperature, behavioural fever and fitness. Proc R Soc BBiol Sci. 2002;269:1599-1607.

15. Lokmer A, Karl MW. Hemolymph microbiome of Pacific oysters in response to temperature, temperature stress and infection. ISME J. 2015;9:670-682.

16. Watts C. The endosome-lysosome pathway and information generation in the immune system. BBA-Proteins Proteomics. 2011;1824:14-21.

17. Wang FY, Yang HS, Gabr HR, Gao F. Immune condition of Apostichopus japonicus during aestivation. Aquaculture. 2008;285:238-243.

18. Zhang W, Huang L, Li J, Li Y, Wei S, Cai L, et al. Transcriptomic analysis of differentially expressed genes in the oviduct of Rhacophorus omeimontis provides insights into foam nest construction. BMC Genomics. 2019;20(1):562.

19. Li W, Mao L, Shu X, Liu R, Hao F, Li J, et al. Transcriptome analysis reveals differential immune related genes expression in bovine viral diarrhea virus-2 infected goat peripheral blood mononuclear cells (PBMCs). BMC Genomics. 2019;20(1):516.

20. Du HX, Bao ZM, Hou R, Wang S, Su HL, Yan JJ, et al. Transcriptome Sequencing and Characterization for the Sea Cucumber Apostichopus japonicus. PLoS One. 2012;7(3):e33311.

21. Huang W, Ye J, Zhang J, Lin Y, He M, Huang J. Transcriptome analysis of Chlorella zofingiensis to identify genes and their expressions involved in astaxanthin and triacylglycerol biosynthesis. Algal Res. 2016;17:236-243.

22. Wang X, Chen C, Gao J, Lv A. Transcriptome profiles in the spleen of African catfish (Clarias gariepinus) challenged with Aeromonas veronii. Fish Shellfish Immunol. 2018;86:858-867

23. Harris M, Deegan J, Ireland A, Lomax J, Ashburner M, Foulger R, et al. The Gene Oncology (GO) database and informatics resource. Nucleic Acids Res. 2004;32:258-261.

24. Ogata H, Goto S, Sato K, Fujibuchi W, Bono H, Kanehisa M. KEGG: kyoto Encyclopedia of Genes and Genomes. Nucleic Acids Res. 1999;27:2934.

25. Finn R, Bateman A, Clements J, Coggill P, Eberhardt R, Eddy SR, et al. Pfam: the protein families database. Nucleic Acids Res. 2013;42(D1):D222-D230.

26. Damian S, Andrea F, Michael K, Milan S, Alexander R, Pablo M, et al. The STRING database in 2011: functional interaction networks of proteins, globally integrated and scored. Nucleic Acids Res. 2010;39 (suppl_1):D561-D568.

27. Zhang X, Sun L, Yuan J, Sun Y, Gao Y, Zhang L, et al. The sea cucumber genome provides insights into morphological evolution and visceral regeneration. PLoS. Biol. 2017;15:e2003790.

28. An ZH, Dong YW, Dong SL. Temperature effects on growth-ration relationships of juvenile sea cucumber Apostichopus japonicus. Aquaculture. 2007;272:644-648.

29. Zhu YY, Li YQ, Xin DD, Chen WR, Shao X, Wang Y, et al. RNA-Seq-based transcriptome analysis of dormant flower buds of Chinese cherry (Prunus pseudocerasus). Gene. 2015;555:362-376.

30. Fei Y, Sun P, Wang JT, Gao QX. Transcriptome analysis of dormant tomonts of the marine fish ectoparasitic ciliate Cryptocaryon irritans under low temperature. Parasites Vectors. 2016;9:280.

31. Ma ZX, Tan YZ, Gui GZ, Feng YG, Cui Q, Song XJ. Transcriptome and gene expression analysis of DHA producer Aurantiochytrium under low temperature conditions. Sci Rep. 2015;5:14446.

32. Wang XW, Zhao XF, Wang JX. C-type Lectin Binds to $\beta$-Integrin to Promote Hemocytic Phagocytosis in an Invertebrate. J Biol Chem. 2014;289(4):2405-2414. 
33. Yamada H, Ohashi E, Abe T, Kusumi N, Li S-A, Yoshida Y, et al. Amphiphysin 1 Is Important for Actin Polymerization during Phagocytosis. Mol Biol Cell. 2007;18:4669-4680.

34. Horwitz M. The Legionnaires' disease bacterium (Legionella pneumophila) inhibits phagosome-lysosome fusion in human monocytes. J Exp Med. 1984;158:2108-2126.

35. Yates R, Hermetter A, Russell D. The kinetics of phagosome maturation as a function of phagosome/lysosome fusion and acquisition of hydrolytic activity. Traffic. 2005;6:413-420.

36. Malik Z, Denning G, Kusner D. Inhibition of Ca signaling by Mycobacterium tuberculosis is associated with reduced phagosome-lysosome fusion and increased survival within human macrophages. J Exp Med. 2000;191:287-302.

37. Garin J, Diez R, Kieffer S, Dermine JF, Duclos S, Gagnon E, et al. The Phagosome Proteome. J Cell Biol. 2001;152(1):165.

38. Sara HA, Jens K, Manuel AG, Claudia H, Markus TB. NSUN6 is a human RNA methyltransferase that catalyzes formation of $\mathrm{m}^{5} \mathrm{C72}$ in specific tRNAs. RNA. 2015;21:1532-1543.

39. Hong J, Sun XZ, Long S, Peng Y, Fang Z, Hui X, et al. Insights into the seasonal adaptive mechanisms of Chinese alligators (Alligator sinensis) from transcriptomic analyses. Aust J Zool. 2018;66:93-102.

40. Kenneth B, Storey JMS. Metabolic rate depression in animals: transcriptional and translational controls. Biol Rev Camb Philos Soc. 2004;79:207-233.

41. The UniProt Consortium. UniProt: A hub for protein information. Nucleic Acids Res. 2014;43:D204-D212.

42. Marc JVDM, Veen BVD, Joost CU, Leemhuis H, Dijkhuizen L. Properties and applications of starch-converting enzymes of the a-amylase family. J Biotechno. 2002;94:137-155.

43. Kong BW, Kim JI, Kim MJ, Kim J. Porcine Pancreatic a-Amylase Hydrolysis of Native Starch Granules as a Function of Granule Surface Area. Biotechnol Prog. 2003;19:1162-1166.

44. Sim L, Quezada CR, Sterchi E, Nichols B, Rose D. Human intestinal maltase-glucoamylase: crystal structure of the n-terminal catalytic subunit and basis of inhibition and substrate specificity. J Mol Biol. 2008;375:782-792.

45. Griffin LD, MacGregor GR, Muzny DM, Harter J, Cook RG, McCabe ER. Synthesis and characterization of a bovine hexokinase 1 cDNA probe by mixed oligonucleotide primed amplification of cDNA using high complexity primer mixtures. Biochem Med Metab Biol. 1989;41(2):125-131.

46. Cipriano D, Wang Y, Bond S, Hinton A, Jefferies K, Qi J, et al. Structure and Regulation of the Vacuolar ATPases. J Bioenerg Biomembr. 2008;1777:599-604.

47. Chu H, Mazmanian S. Innate immune recognition of the microbiota promotes host-microbial symbiosis. Nat Immunol. 2013;14:668-675.

48. Ezekowitz R, Sastry K, Bailly P, Warner A. Molecular characterization of the human macrophage mannose receptor: demonstration of multiple carbohydrate recognition-like domains and phagocytosis of yeasts in Cos-1 cells. J Exp Med. 1991;172:1785-1794.

49. Marttila IF, Turja R, Lehti M, Karikoski M, Maksimow M, Niemelä J, et al. Macrophage mannose receptor on lymphatics controls cell trafficking Blood. 2008;112:64-72.

50. Insall R, Müller TA, Machesky L, Köhler J, Simmeth E, Atkinson S, et al. Dynamics of the Dictyostelium Arp2/3 complex in endocytosis, cytokinesis, and chemotaxis. Cell Motil Cytoskel. 2001;50:115-128.

51. Trautwein M, Dengjel J, Schirle M, Spang A. Arf1p provides an unexpected link between COPI vesicles and mRNA in Saccharomyces cerevisiae. Mol Biol Cell. 2004;15:5021-5037.

52. Derrien V, Couillault C, Franco M, Martineau S, Montcourrier P, Houlgatte R, et al. A conserved C-terminal domain of EFA6-family ARF6-guanine nucleotide exchange factors induces lengthening of microvilli-like membrane protrusions. J Cell Sci. 2002;115:2867-2879.

53. Pianta A, Arvikar S, Strle K, Drouin E, Wang Q, Costello C, et al. Two rheumatoid arthritis-specific autoantigens correlate microbial immunity with autoimmune responses in joints. J Clin Invest. 2017;127(8):2946-2956.

54. Barth H, Schnober E, Zhang E, Linhardt R, Depla E, Boson B, et al. Viral and cellular determinants of hepatitis C virus envelope-heparan sulfate interaction. J Hepatol. 2006;80(21):10579-1059.

55. Mok A, Cao H, Hegele RA. Genomic basis of mucopolysaccharidosis type IIID (MIM 252940) revealed by sequencing of GNS encoding Nacetylglucosamine-6-sulfatase. Genomics. 2003;81(1):1-5.

56. Wudunn D, Spear P. Initial interaction of Herpes simplex virus with cells is binding to heparan sulfate. J Virol. 1989;63:52-58.

57. Freeman C, Hopwood J. Lysosomal Degradation of Heparin and Heparan Sulphate. Adv Exp Med Biol. 1992;313:121-134.

58. Im J. Comparative transcriptomics reveals CrebA as a novel regulator of infection tolerance in D. melanogaster. PLoS Pathog. 2018;14(2):e1006847.

59. Izai K, Uchida Y, Orii T, Yamamoto S, Hashimoto T. Novel Fatty acid beta-oxidation enzymes in rat liver mitochondria. I. Purification and properties of very-long-chain acyl-coenzyme A dehydrogenase. J Biol Chem. 1992;267:1027-1033.

60. Ikeda Y, Okamura-Ikeda K, Tanaka K. Purification and characterization of short-chain, medium-chain, and long-chain acyl-CoA dehydrogenases from rat liver mitochondria. J Biol Chem. 1985;260:1311-1325.

Page $10 / 18$ 
61. Page G, Hayworth K, Wade R, Harris A, Bureau D. Non-specific immunity parameters and the formation of advanced glycosylation end-products (AGE) in rainbow trout, Oncorhynchus mykiss (Walbaum), fed high levels of dietary carbohydrates. Aquac Res.1999;30:287-297.

62. Argayosa A, Bernal RA, Luczon A, Arboleda J. Characterization of mannose-binding protein isolated from the African catfish (Clarias gariepinus B.) serum. Aquaculture. 2011;310:274-280.

\section{Tables}

Table 1 . The transcriptome summary of $A$. japonicus.

\begin{tabular}{|c|c|c|c|c|c|c|c|c|c|}
\hline Sample & D1 & D2 & D3 & D4 & D5 & D6 & R1 & $\mathrm{R} 2$ & R3 \\
\hline $\begin{array}{l}\text { Raw } \\
\text { reads }\end{array}$ & 50602640 & 56679926 & 50018434 & 57880594 & 45709584 & 51717568 & 54319176 & 56110888 & 51838102 \\
\hline $\begin{array}{l}\text { Clean } \\
\text { reads }\end{array}$ & 48571520 & 54600842 & 48289312 & 55666196 & 43871734 & 49740432 & 52256230 & 53770364 & 50456992 \\
\hline $\begin{array}{l}\text { Clean } \\
\text { bases }\end{array}$ & $7.29 \mathrm{G}$ & 8.19G & $7.24 \mathrm{G}$ & $8.35 G$ & $6.58 \mathrm{G}$ & $7.46 \mathrm{G}$ & $7.84 \mathrm{G}$ & 8.07G & $7.57 \mathrm{G}$ \\
\hline $\begin{array}{l}\text { Error } \\
\text { rate }\end{array}$ & 0.03 & 0.03 & 0.03 & 0.03 & 0.03 & 0.03 & 0.03 & 0.03 & 0.03 \\
\hline Q20 & 97.44 & 97.37 & 97.54 & 97.43 & 97.35 & 97.47 & 97.5 & 97.31 & 97.49 \\
\hline Q30 & 92.9 & 92.83 & 93.14 & 92.88 & 92.7 & 92.98 & 93.02 & 92.62 & 93 \\
\hline GC(\%) & 41.12 & 41.15 & 41.31 & 41.71 & 41.02 & 41.19 & 41.54 & 40.57 & 41.04 \\
\hline $\begin{array}{l}\text { Total } \\
\text { map }\end{array}$ & $31584861(65.03 \%)$ & $34219051(62.67 \%)$ & $31264585(64.74 \%)$ & $37607631(67.56 \%)$ & $29672888(67.64 \%)$ & $32753242(65.85 \%)$ & $35855693(68.62 \%)$ & $35576765(66.16 \%)$ & $32801415(65.01 \%)$ \\
\hline $\begin{array}{l}\text { Unique } \\
\text { map }\end{array}$ & $29363445(60.45 \%)$ & $31904575(58.43 \%)$ & $29141658(60.35 \%)$ & $34832088(62.57 \%)$ & $27534777(62.76 \%)$ & $30347259(61.01 \%)$ & $33111122(63.36 \%)$ & $32984974(61.34 \%)$ & $30501515(60.45 \%)$ \\
\hline $\begin{array}{l}\text { Multi } \\
\text { map }\end{array}$ & $2221416(4.57 \%)$ & $2314476(4.24 \%)$ & $2122927(4.4 \%)$ & 2775543(4.99\%) & $2138111(4.87 \%)$ & $2405983(4.84 \%)$ & $2744571(5.25 \%)$ & $2591791(4.82 \%)$ & $2299900(4.56 \%)$ \\
\hline $\begin{array}{c}\text { read1 } \\
\text { map }\end{array}$ & $14772247(30.41 \%)$ & $16053234(29.4 \%)$ & $14639063(30.32 \%)$ & $17533733(31.5 \%)$ & $13868463(31.61 \%)$ & $15260800(30.68 \%)$ & 16642194(31.85\%) & $16616277(30.9 \%)$ & $15339727(30.4 \%)$ \\
\hline $\begin{array}{c}\text { read2 } \\
\text { map }\end{array}$ & $14591198(30.04 \%)$ & $15851341(29.03 \%)$ & $14502595(30.03 \%)$ & 17298355(31.08\%) & $13666314(31.15 \%)$ & $15086459(30.33 \%)$ & $16468928(31.52 \%)$ & $16368697(30.44 \%)$ & $15161788(30.05 \%)$ \\
\hline $\begin{array}{c}\text { Positive } \\
\text { map }\end{array}$ & 14669856(30.2\%) & $15940367(29.19 \%)$ & $14564607(30.16 \%)$ & $17433457(31.32 \%)$ & $13766965(31.38 \%)$ & 15178136(30.51\%) & $16557296(31.68 \%)$ & $16492514(30.67 \%)$ & $15247913(30.22 \%)$ \\
\hline $\begin{array}{c}\text { Negative } \\
\text { map }\end{array}$ & $14693589(30.25 \%)$ & $15964208(29.24 \%)$ & $14577051(30.19 \%)$ & 17398631(31.26\%) & $13767812(31.38 \%)$ & $15169123(30.5 \%)$ & 16553826(31.68\%) & $16492460(30.67 \%)$ & $15253602(30.23 \%)$ \\
\hline $\begin{array}{c}\text { Splice } \\
\text { map }\end{array}$ & $11406389(23.48 \%)$ & $12867289(23.57 \%)$ & $11561477(23.94 \%)$ & $14199005(25.51 \%)$ & $10420305(23.75 \%)$ & 11880956(23.89\%) & $13676444(26.17 \%)$ & $12081880(22.47 \%)$ & $11860245(23.51 \%)$ \\
\hline $\begin{array}{l}\text { Unsplice } \\
\text { map }\end{array}$ & $17957056(36.97 \%)$ & $19037286(34.87 \%)$ & $17580181(36.41 \%)$ & $20633083(37.07 \%)$ & $17114472(39.01 \%)$ & $18466303(37.13 \%)$ & $19434678(37.19 \%)$ & 20903094(38.87\%) & $18641270(36.94 \%)$ \\
\hline $\begin{array}{c}\text { Proper } \\
\text { map }\end{array}$ & $25504410(52.51 \%)$ & $27566326(50.49 \%)$ & $25217040(52.22 \%)$ & $30396214(54.6 \%)$ & $23950864(54.59 \%)$ & $26391288(53.06 \%)$ & $28966836(55.43 \%)$ & $28637800(53.26 \%)$ & $26581470(52.68 \%)$ \\
\hline
\end{tabular}

Table 2. DEGs involved incarbohydrate metabolism, fatty acid metabolism, TCA circle and phagocytosis pathways in dormant A.japonicus. 


\begin{tabular}{|c|c|c|c|c|}
\hline Gene ID & KEGG annotation & Expression & $\log _{2}$ FoldChange (TPM) & Gene description \\
\hline novel.15692 & Endocytosis & Up & 1.46 & Actin-related protein 2/3 complex subunit 5A (ARPC5A) \\
\hline novel.15774 & Endocytosis & Up & 2.31 & Actin-related protein $2 / 3$ complex subunit 5B (ARPC5B) \\
\hline novel.14744 & Endocytosis & Up & 1.11 & PH and SEC7 domain-containing protein (PSD) \\
\hline novel.9544 & Lysosome & Up & 1.18 & N-acetylglucosamine-6-sulfatase (GNS) \\
\hline gene15922 & Lysosome & Up & 2.99 & N-acetylgalactosamine-6-sulfatase-like (GNSL) \\
\hline gene25464 & Phagosome/Lysosome & Up & 2.06 & V-type $\mathrm{H}^{+}$-transporting ATPase subunit A (VATPA) \\
\hline novel.2546 & Phagosome & Up & 3.3 & Macrophage mannose receptor 1 (MRC1) \\
\hline gene7968 & Starch and sucrose metabolism & Up & 1.28 & Hexokinase-1 (HK1) \\
\hline gene2722 & Starch and sucrose metabolism & Up & 7.9 & Pancreatic alpha-amylase (PAMY) \\
\hline gene2721 & Starch and sucrose metabolism & Up & $>10$ & Alpha-amylase B (AMYB) \\
\hline gene10557 & Starch and sucrose metabolism & Up & 9.92 & Alpha-amylase (AMY) \\
\hline gene1069 & Starch and sucrose metabolism & Up & 6.33 & Maltase-glucoamylase (MGAM) \\
\hline gene22358 & Fatty acid metabolism & Up & 2.86 & Fatty acid synthase (FASN) \\
\hline gene22359 & Fatty acid metabolism & Up & 3.15 & Fatty acid synthase-like (FASNL) \\
\hline gene10974 & Fatty acid metabolism & Up & 1.13 & Very long-chain specific acyl-CoA dehydrogenase (ACADVL) \\
\hline gene8465 & Fatty acid metabolism & Up & 2.83 & Long-chain specific acyl-CoA dehydrogenase (ACADL) \\
\hline gene13076 & Fatty acid metabolism & Up & 1.23 & Malonyl-CoA-acyl carrier protein transacylase (MCAT) \\
\hline gene11165 & NA & Up & 3.63 & Ubiquitin $\mathrm{C}$ variant (UBCV) \\
\hline
\end{tabular}

Table 3. Sequences of primers used in this study. 


\begin{tabular}{|c|c|}
\hline Primers & Sequence $\left(5^{\prime}\right.$ to $\left.3^{\prime}\right)$ \\
\hline \multicolumn{2}{|l|}{ qRT-PCR } \\
\hline ARP-R & GAAGGAGGACATGGCTCGTAA \\
\hline \multicolumn{2}{|c|}{ ARPC5A-F GTTGAAGAAACACAAGGCGGT } \\
\hline \multicolumn{2}{|c|}{ ARPC5B-R CCTTCTCAACTTCGCTGGCTT } \\
\hline \multicolumn{2}{|c|}{ ARPC5B-F AAGAAACACAAGGCGGTCCA } \\
\hline PSD-R & GAAGAAGTGGTGCCCCAGATA \\
\hline PSD-F & CAGGCAATCAAGGCAGAACC \\
\hline GNS-R & CTGGAAGGGATCGTGTGTCA \\
\hline GNS-F & СТСТССССАACAGACAACTACAT \\
\hline GNSL-R & ATCGTGTTCTTCTTCAATGTGGC \\
\hline GNSL-F & AGGAACCTGCTACCGTTATGGA \\
\hline VATPA-R & TTCGTCTTTGTCCGCATCGT \\
\hline VATPA-F & CAGCATTTCTTTGACGAGGTAGC \\
\hline MRC1-R & CACCACGCCGTTTACATTCAC \\
\hline MRC1-F & AACACCTGGGAGTTTTCTTTCG \\
\hline HK1-R & TGTTTATGATGGTCTGTTTTGGCT \\
\hline HK1-F & TACGGGAACGCTGATGTCTTG \\
\hline PAMY-R & CCCATTTGGATTTGGACACCTG \\
\hline PAMY-F & TCGGTGTTCGCATTTATGTTGAT \\
\hline AMYB-R & TCGTAAGCAAATCTTTCGCACC \\
\hline AMYB-F & TCTGTTTGAATGGAAGTGGTCTGA \\
\hline AMY-R & TGGTCCCTATCGGAGAAAGCA \\
\hline AMY-F & TTTGGTGAAGGTTGGGGTTTTAT \\
\hline MGAM-R & CCAGTTACCTCTTCTGCCCC \\
\hline MGAM-F & САСТСТСТTCTACСАCGCCAAT \\
\hline FASN-R & ATGAACTTTGACCTCCGCCT \\
\hline FASN-F & GTAGCGACGGAGATGGCAC \\
\hline FASNL-R & CCACACGATTGGTTAGGCTG \\
\hline FASNL-F & AATGGTGGTTATGTCGGGCT \\
\hline ACADVL-R & RTACCCTTGAAGCCGATGGAC \\
\hline ACADVL-F & AGGTCTC \\
\hline ACADL-R & GTAACCCTGCСТСТССТССТАA \\
\hline ACADL-F & CACTAATGGCTACCTCGCTGAT \\
\hline MCAT-R & CAACCGCAGCCAGTGATGTC \\
\hline MCAT-F & TGTTGGTATGTGTGAGGCGTTC \\
\hline UBCV-R & TCTAGCAGGATTGGTTGGAGC \\
\hline UBCV-F & AGGGAGTTGGAAAAAGAGAAGTCA \\
\hline$\beta$-actin-R & TTGTGGTAAAGGTGTAGCCTCTCTC \\
\hline$\beta$-actin-F & TTATGCTCTTCCTCACGCTATCC \\
\hline
\end{tabular}

\section{Additional File Legend}

Addtional file 1: Table S1. Characterization of genes in PPI analysis

\section{Figures}




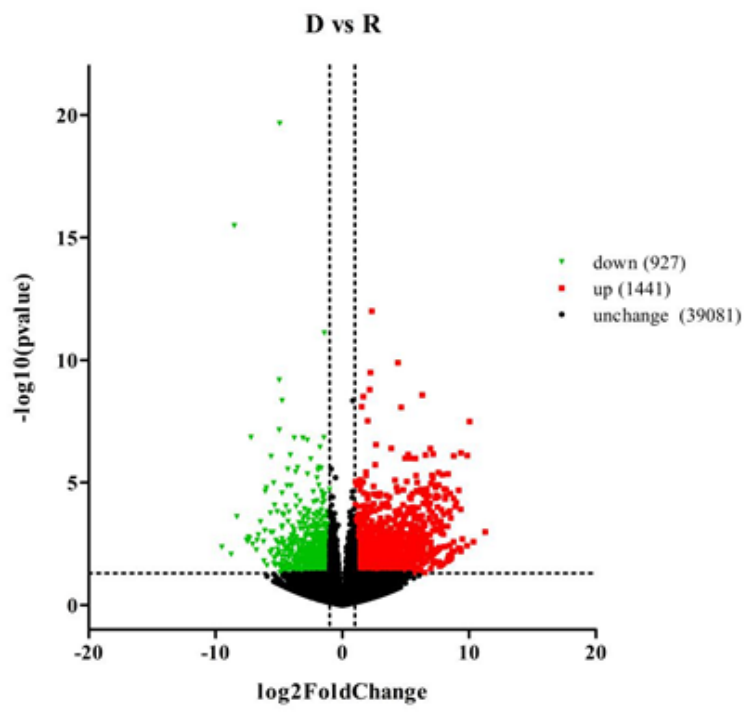

Figure 1

Volcano plot of differently expressed genes (DEGs) between the dormant group (D) and revival group (R).

A

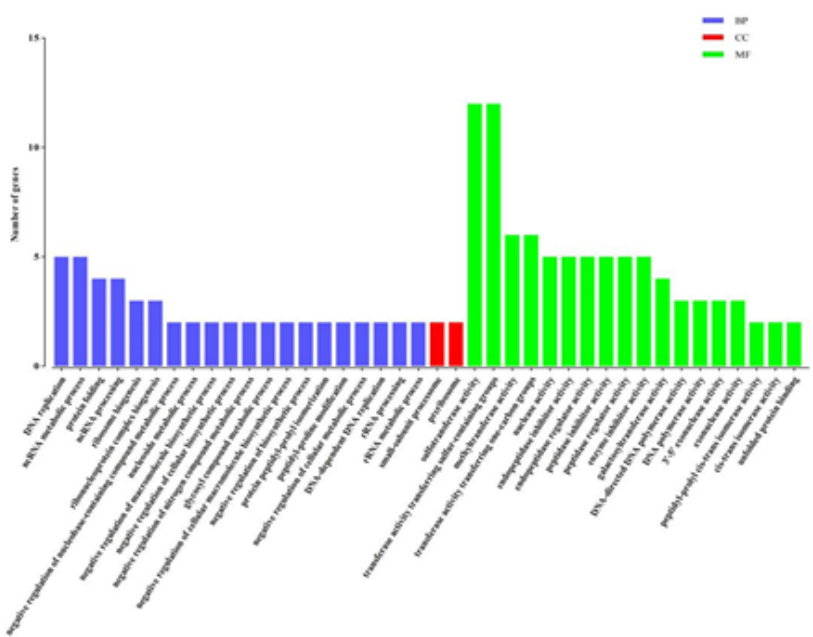

B

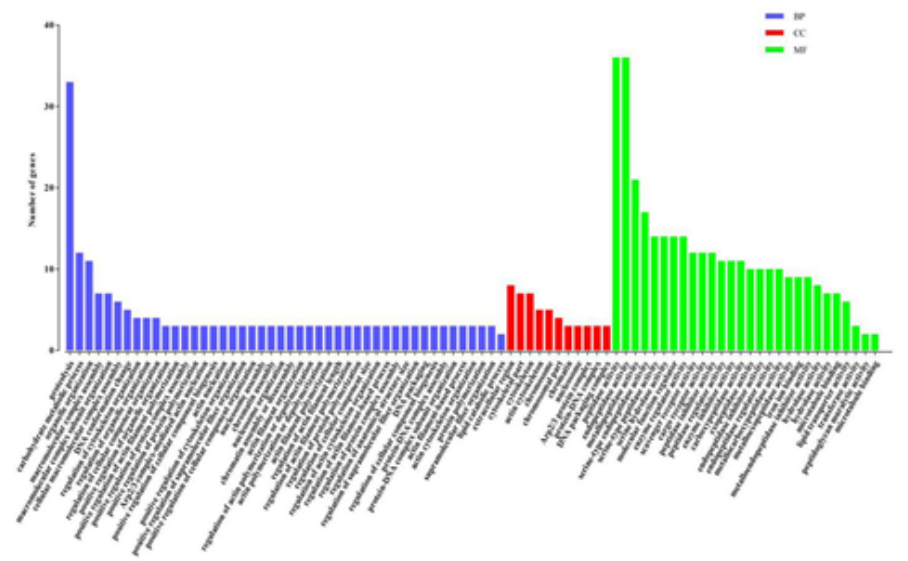

Figure 2

Gene Ontology (GO) annotated for differently expressed genes (DEGs) at the levels of Biological process (BP), Cellular component (CC) and Molecular function (MF). (A) and (B) represented down-regulated and up-regulated genes in the dormant group, respectively. 
A

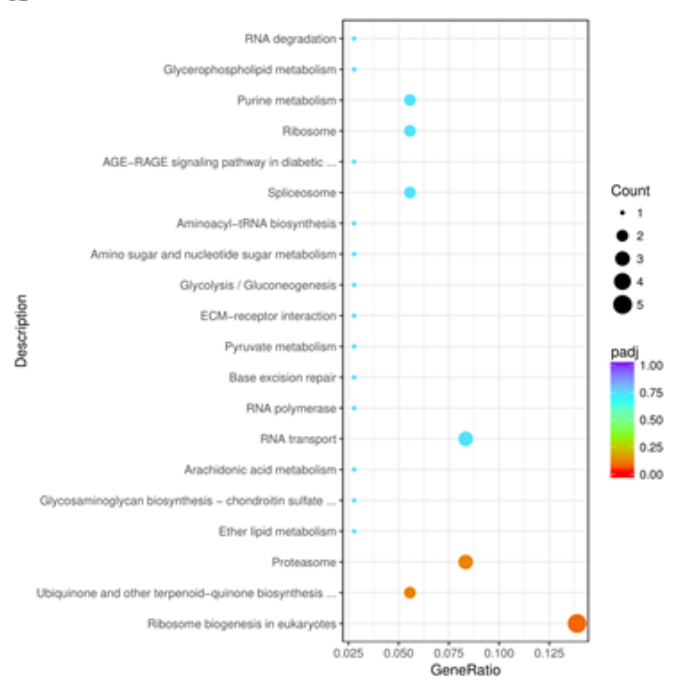

B

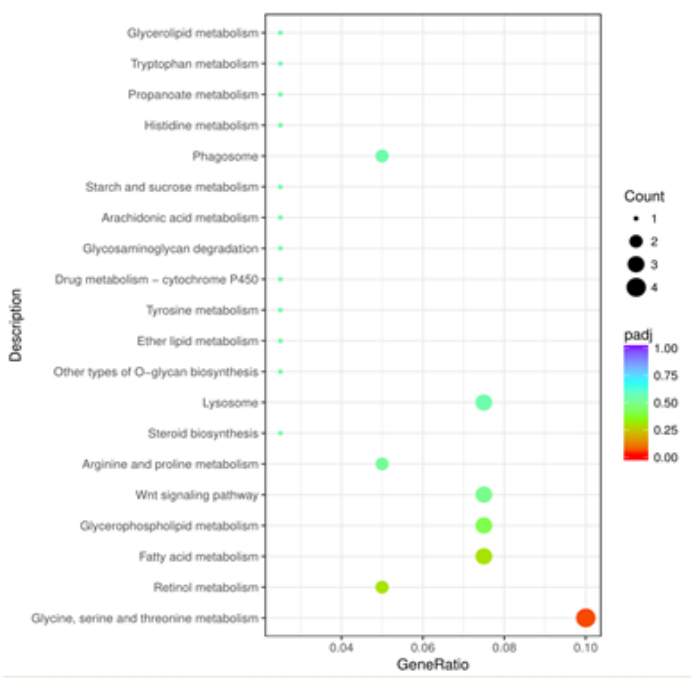

Figure 3

KEGG annotation of differently expressed genes (DEGs) between the dormant group (D) and revival group (R). (A) and (B) represented downregulated and up-regulated genes in dormant group, respectively.

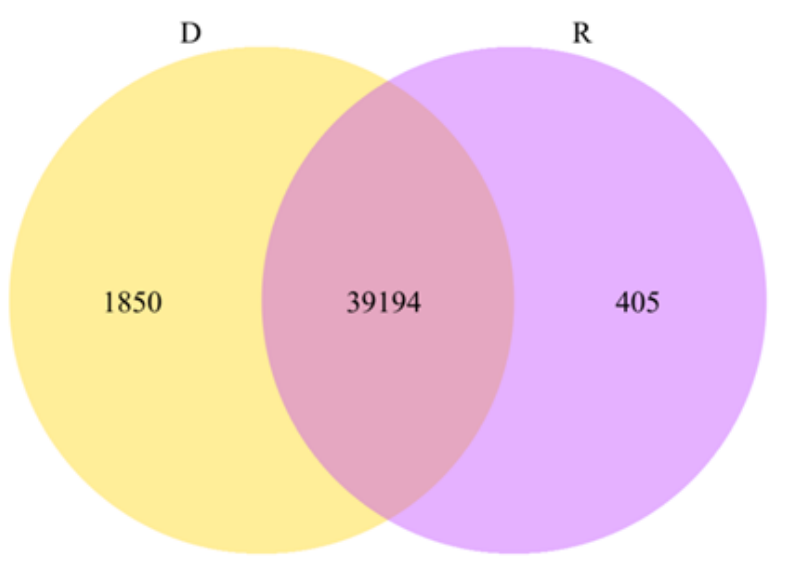

Figure 4

Venn diagram showing the number of differently expressed genes (DEGs) between the dormant group (D) and revival group (R). 


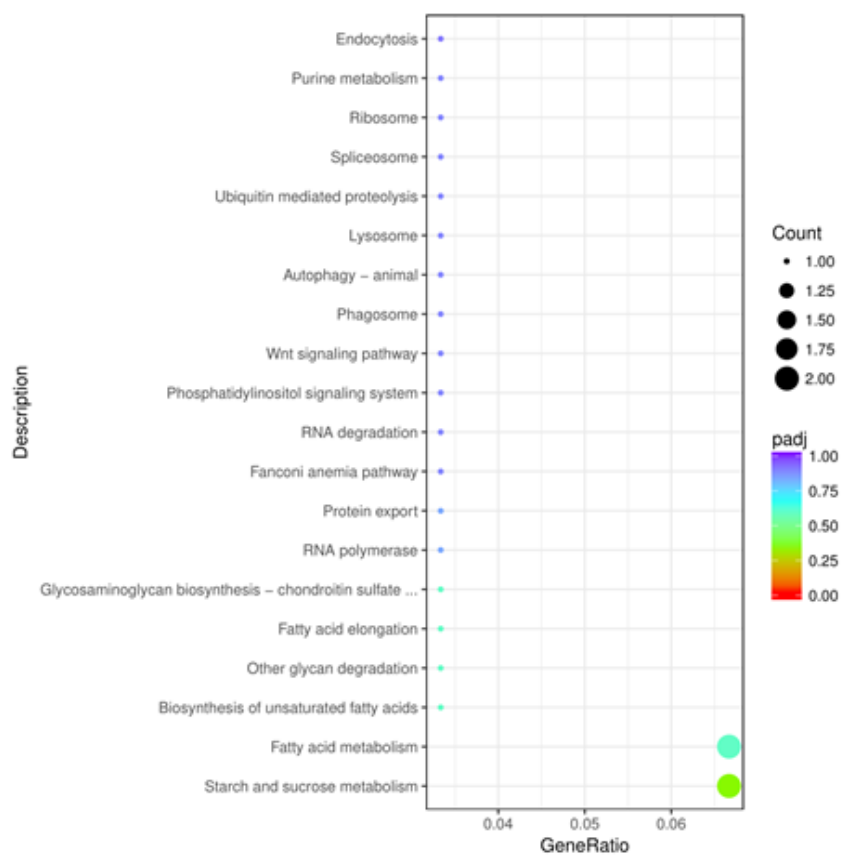

Figure 5

KEGG annotation of exclusively expressed genes in the dormant (D) and revival group (R). 
A

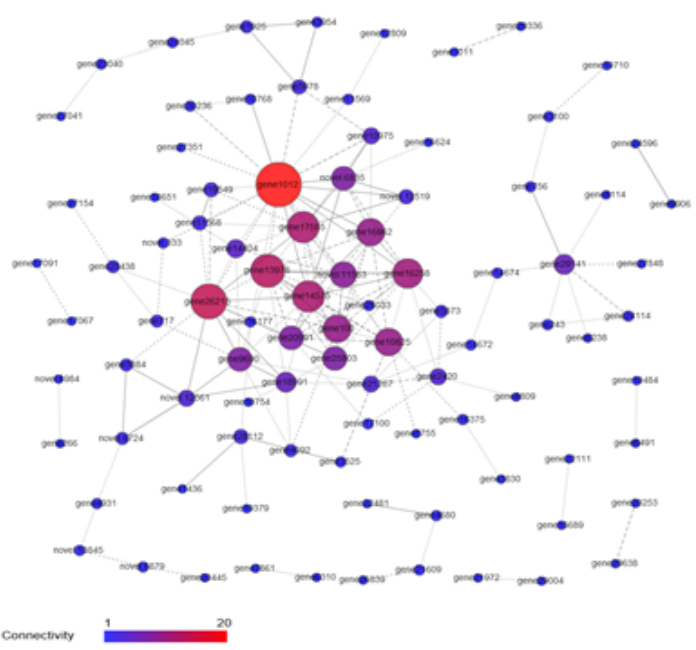

B

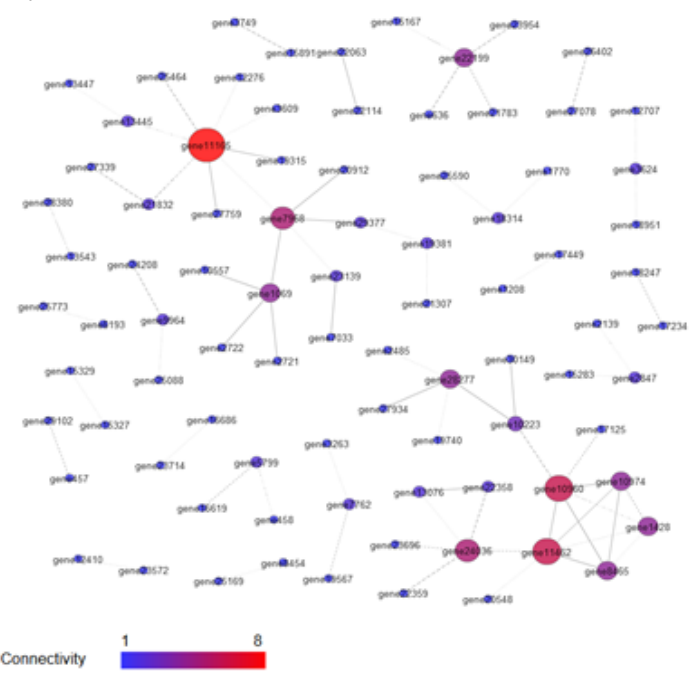

\section{Figure 6}

PPI network of differently expressed genes (DEGs) based on STING analysis results. (A) and (B) represented down-regulated and up-regulated genes in the dormant group, respectively. The size and color of the node indicate the connectivity.

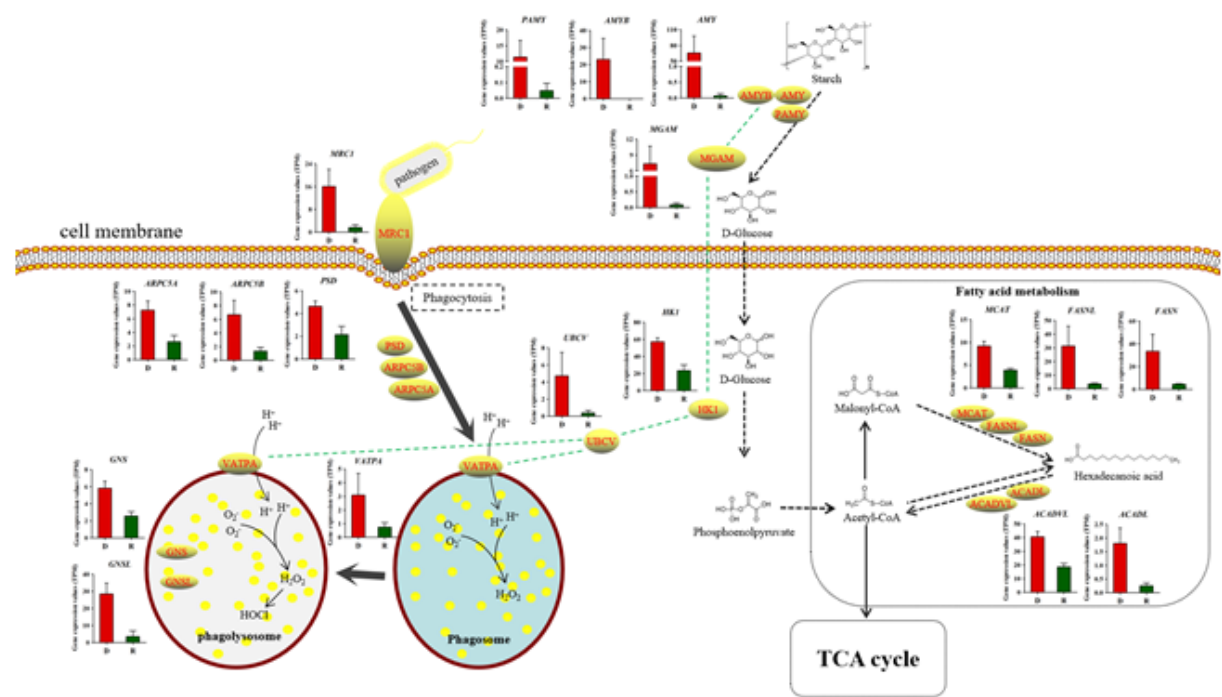

Figure 7 
Genes and their RNA-seq expression values in TPM (transcripts per million) involved in carbohydrate metabolism, fatty acid metabolism, TCA circle and phagocytosis pathways of dormant A. japonicus. The black solid arrow represents direct conversion between two substances; the black dotted arrow represents indirect conversion between two substances, that some intermediate products were omitted; the green dotted line represents the interactions between two proteins.

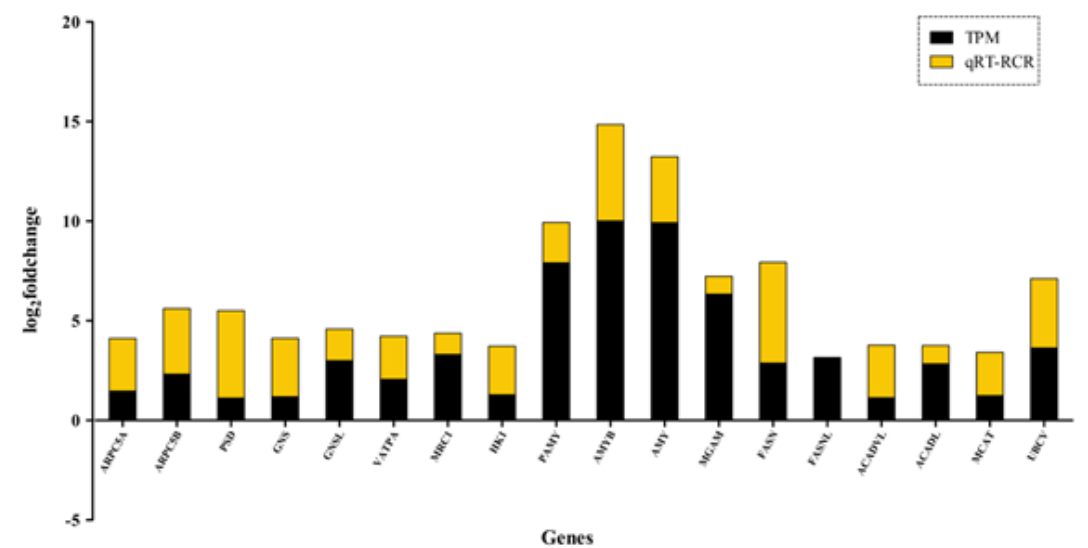

\section{Figure 8}

Eighteen genes of pathways analysis in Fig. 7 were validated by comparison of RNA-Seq (TPM) and qRT-PCR. The yellow bars represent the results of qRT-PCR, and black bars represent TPM value.

\section{Supplementary Files}

This is a list of supplementary files associated with this preprint. Click to download.

- Additionalfiles1.doc 